\title{
TRANSITION BETWEEN FLAT MAGNITUDE AND FLAT GROUP DELAY LOW PASS RECURSIVE DIGITAL FILTERS
}

\author{
NEGOVAN STAMENKOVIĆ ${ }^{1, \star}$, MILAN DEJANOVIĆ ${ }^{1}$ \\ ${ }^{1}$ Faculty of Natural Sciences and Mathematics, University of Priština, Kosovska Mitrovica, Serbia
}

\begin{abstract}
Many a paper has been written on the characteristics of the continuous-time and discrete-time low-pass filters which are designed to have either maximally flat magnitude characteristics or maximally flat group delay responses. The polynomial (all pole) low pass recursive digital filters which are characterised by characterise the transition between a flat magnitude and flat group delay characteristics, named Transitional Butterworth Thiran (TBTh) recursive digital filters, is considered. The characteristics of the resulting filter change gradually from the characteristics of the well known Butterwort's filter to the characteristics of the Thiran's filter with the maximally flat group delay characteristic. Poles of the TBTh filter are obtained by interpolating between poles Butterworth and Thiran's filters by varying a parametar which controls the pole positions that enables a tradeoff between the steepness magnitude characteristic at passband edge and group delay deviation.
\end{abstract}

Keywords: Transitional filters, IIR filter, Butterworth filter, Thiran filter, Group delay.

\section{INTRODUCTION}

In the field of circuits and system theory, transitional filters combine the frequency response of two different filters (typically stopband attenuation and constant group delay types) to achieve a compromise between attenuation and group delay characteristics. There are generally two types of transitional filters: transitional filters combining filter poles and transitional filters combining classical orthogonal polynomials.

Combining filter poles by means of an interpolation formula results in transitional characteristics. In other words, the transitional $\mathrm{AB}$ (TAB) filters having characteristics that tranzition smoothly from those of filter A to those of filter B as a design parameter $m$ is varied from zero to one. The transition is accomplished using the method which have been described of Peless \& Murakami (1957), which have found each pole location of the TBT transfer function in the complex frequency plane. They were to change the transfer function smoothly from that of the Butterworth to those of the Thomson filters by varying a parameter which controls the pole positions. Although a large variety of filter specifications can be satisfied by an appropriate choice of the variabile parametar, this class of filters offers no advantages over Butterworth or Thomson filters in respect of steady-state and transient responses. Other particular method will operate directly on the filter poles (Aiello \& Angelo, 1974) is transitional LegendreThomson (TLT) whose frequency characteristics can be approximated in a maximum cutoff rate (Papoulis, 1958) (Legendre) or in a maximally flat delay sense (Thomson).

In the paper Johnson et al. (1979) have considered transitional rational filters using the interpolation method of Peless and Murakami to locate both the zeros and poles of the transition fil- ter. The transitional rational $\mathrm{AB}$ filters are obtained by finding each pole (zero) of the TRAB filter as an interpolation between a pole (zero) of the A filter and a corresponding pole (zero) of the B filter. In particular example, A filter is well known inverse Chebyshev filter, and B filter is Bessel rational filter (Johnson et al., 1976).

Other allpole monotonic filter functions with a mentioned variable parameter $m$ that enables a tradeoff between the maximum permissible overshoot and the rise time in the time domain, so that a large variety of filter specifications can be met in practical design with a single class of filter functions, are transitional Halpern-Thomson filters (Lazović \& Radmanović, 1975).

These filters share the fact that the transition operation is performed in the complex s-plane with pole locations bounded by the two filter responses and they are not suitable for mapping into discrete time domain.

The mixture of two classical orthogonal polynomials to generate a new polynomial is an alternative means of generating transitional filters. These comprise the well-known transitional Butterworth- Chebyshev (TBC) filter, a mixture of Butterworth and Chebyshev components (Budak \& Aronhime, 1971; Thajchayapong et al., 1978; Roy \& Varanasi, 1978). Transitional ultraspherical-ultraspherical (TUU) filters as a generalization of the transitional Butterworth-Chebyshev fites is presented in the paper Attikiouzel \& Dang (1978).

In addition to the transient filters with simple poles, transitional filters with multiple poles proposed in the papers Stojanović \& Pavlović (1979) and Stojanović \& Pavlović (1980). These allpole functions, based on the Butterwortth and Chebyshev filters, have been derived by numerical optimization of the magnitude 
passband response under the constraint of a double or higher-order multiplicity of the dominant pair of poles.

A technique commonly used in the design of recursive digital filters is the use of bilinear transformation of continuous-time filter transfer functions. Moreover, such filters are not the true classical counterparts in the digital domain. A method for direct computing the poles of transitional all-pole Butterworth-Chebyshev recursive digital filters is presented in the papers Nikolić \& Stojanović (1996); Stojanović \& V. Nikolić (1998); Stojanović \& Nikolić (1993). These filters offer a gradual transition between Butterworth and Chebyshev filters. Number of transitional filter is $n$, where $n$ is the degree of transitional filters.

The main object of this paprr is to introduce a new class of discrete-time allpole filters with a variable parameter that enables a tradeoff between the maximally flat passband attenuation (Butterworth) and the maximally flat group delay (Thiran), so that a large variety of filter specifications can be met in practical design with a single class of filter functions.

These linear time-Invariant digital filters, referred to as transitional Butterworth-Thiran (TBTh) filters, to different from TBT filtes, provide considerably trade off between constant group delay and attenuation characteristic. The transfer function of certain TBTh filters has been computed, tabulated and the figures clearly illystrate the transitional nature of these filters.

\section{SHORT REVIEW OF THE BUTTERWORTH ALLPOLE IIR DIGITAL FILTERS}

The general form of the squared magnitude characteristic, both in continuous-time and discrete-time domain, of a lowpass Butterworth filter wityh no transmission zeros (Stojanović et al., 2014) may be expressed as

$$
\left|H_{B}\left(x^{2}\right)\right|^{2}=\frac{1}{1+\varepsilon^{2} x^{2 n}},
$$

where $n$ is filter degree, $x$ is frequency variable and $\varepsilon$ is is a design parameter that controls the passband loss, known as the passband edge ripple factor, which is related to the maximal passband attenuation $a_{\max }$ (in $\mathrm{dB}$ ), as evidenced by $\varepsilon=\sqrt{10^{0.1 a_{\max }}-1}$. Without loss of generality, $\varepsilon^{2}=1$ can be substituted in (1), then the maximum passband attenuation is specified as $a_{\max }=3 \mathrm{~dB}$.

If frequency variable $x$ is continuous-time angular frequency, $x^{2}=-s^{2}$, then function (1) is the magnitude characteristic of the continuous-time low-pass Butterworth transfer function, because $s=j \omega$ at real frequencies.

On the other hand, the discrete-time polynomial filter is designed from the magnitude function (1) by a technique known as analytic continuation, trough substitution (Stamenković \& Stojanović, 2014)

$$
x^{2} \mapsto \frac{\sin ^{2} \frac{\omega T}{2}}{\sin ^{2} \frac{\omega_{c} T}{2}}=-\left.\frac{(z-1)^{2}}{(2 \alpha)^{2} z}\right|_{z=e^{j \omega T}},
$$

where $\omega$ is continuous-time angular frequency, $\omega_{c}$ is the $3-\mathrm{dB}$ cutoff frequency, $\alpha=\sin \left(\omega_{c} T / 2\right)$ and $T$ is sampling period, which shows that this transformation leads to the transfer function with multiple zero at the origin in the $z$-plane. Substituting (5) into (1) the discrete-time magnitude squared function $H_{B}(z) H_{B}(1 / z) H_{B}(z) H_{B}(1 / z)$ of allpole lowpass Butterworth filter is obtained as folloes

$$
H_{B}(z) H_{B}\left(\frac{1}{z}\right)=\frac{z^{n}}{d_{0}+d_{1} z+\cdots+d_{1} z^{2 n-1}+d_{0} z^{2 n}},
$$

where

$$
d_{i}= \begin{cases}(-1)^{i+n} \varepsilon\left(\begin{array}{c}
2 n \\
i
\end{array}\right) \frac{1}{4 \alpha^{n}}, & \text { za } i=0,1, \ldots, n-1, \\
\varepsilon\left(\begin{array}{c}
2 n \\
n
\end{array}\right) \frac{1}{4 \alpha^{n}}+1, & \text { za } i=n .\end{cases}
$$

are the first $n+1$ coefficients $d_{i}$ in the closed form of the mirrorimage polynomials.

By equating the denominator of (3) with zero, roots will occur in the mirror-image pairs. Poles of the polynomial IIR Butterworth filters $H_{B}(z)$ are merely roots lying inside the unit circle $p_{i}$, hence the resulting filter is stable. Thus

$$
H_{B}\left(z^{-1}\right)=\frac{h_{0}}{z^{-n}+a_{2} z^{-n+1}+\cdots+a_{n} z^{-1}+a_{n+1}},
$$

where $h_{0}=\sum_{i=1}^{n+1} a_{i}$ is a constant that ensures that amplitude $\left|H_{B}(\omega)\right|$ is bounded above by unity.

\section{IIR DIGITAL FILTERS WITH MAXIMALLY FLAT GROUP DELAY}

Thiran (1971) developed an analytical method for deriving the all pole transfer function of the IIR digital filter that approximates a constant group delay in the maximally flat sense. Let $\tau$ be the prescribed group delay, and the allpole transfer function be chosen in the closed form as

$$
H_{T}\left(z^{-1}\right)=\frac{\frac{2 n !}{n !} \frac{1}{\prod_{i=n+1}^{2 n}(2 \tau+i)}}{\sum_{k=0}^{n}(-1)^{k}\left(\begin{array}{l}
n \\
k
\end{array}\right) \prod_{i=0}^{n} \frac{2 \tau+i}{2 \tau+k+i} z^{-k}}=\frac{\sum_{i=0}^{n} a_{i}}{\sum_{i=0}^{n} a_{i} z^{-i}},
$$

whose gain is adjusted to unity at $z^{-1}=1$.

The group delay $\tau_{T}\left(z^{-1}\right)$ of the low-pass filter $H_{T}\left(z^{-1}\right)$ is mirror-image, rational function in $z$, that can be easily calculated by employing the following formula

$$
\begin{aligned}
\tau_{T}\left(z^{-1}\right) & =-\frac{1}{2}\left[\frac{z}{\left.H_{T}\left(z^{-1}\right)\right)} \frac{\mathrm{H}_{T}\left(z^{-1}\right)}{\mathrm{Z}}-\frac{z}{H_{T}(z)} \frac{\mathrm{H}_{T}(z)}{\mathrm{Z}}\right] \\
& =\frac{1}{2}\left[\frac{\sum_{i=1}^{n} i a_{i} z^{-i}}{\sum_{i=0}^{n} a_{i} z^{-i}}+\frac{\sum_{i=1}^{n} i a_{i} z^{-n+i}}{\sum_{i=0}^{n} z^{-n+i}}\right]
\end{aligned}
$$

For a filter of degree $n$ and with a maximally flat group delay the first $n-1$ derivatives of the group delay with respect to $z^{2}$ are zero at $z=1$. 
Using (7), it can be derived an expression for the group delay of transfer function (5) or (6) at $z^{-1}=1(\omega=0)$ as (Stamenković et al., 2018)

$$
\tau_{T}(1)=-\frac{\sum_{i=0}^{n} i a_{i}}{\sum_{i=0}^{n} a_{i}}
$$

and at $z^{-1}=-1(\omega=\pi)$ as

$$
\tau_{T}(-1)=-\frac{\sum_{i=0}^{n} i(-1)^{i+1} a_{i}}{\sum_{i=0}^{n}(-1)^{i+1} a_{i}}
$$

Equations (8) and (9) are valid for all polynomial filters.

Thiran has also shown that the above transfer function is stable for all finite positive values of $\tau$. Using the above formula, the coefficients of the denominator polynomial of $H_{T}\left(z^{-1}\right)$ for $n=$ 8 (and $\tau=2$ ) are tabulated in Table 1 .

\section{TRANSFER FUNCTION OF THE TRANSITIONAL FIL- TER}

The poles position of the transitional Butterworth-Thiran transfer function is determined by defining a real parameter $m$ that provides a linear variation in the phasorangle of the pole in a relation involving the corresponding Butterworth and Thiran poles

$$
s_{k}=s_{k B}^{1-m} s_{k T}^{m}, \text { for } 0 \leq m \leq 1
$$

where $s_{k}$ is the generical pole of the TBTh transfer function, $s_{k B}$ and $s_{k T}$, the Butterworth and Thiran poles, respectively. The position of $s_{k}$ varies smoothly, as $m$ increases, from $s_{k}=s_{k B}$ for $m=0$, to $s_{k}=s_{k T}$ for $m=1$. The magnitude and the argument of $s_{k}$ are

$$
\begin{aligned}
\left|s_{k}\right| & =\left|s_{k B}\right|^{1-m}\left|s_{k T}\right|^{m} \\
\arg \left(s_{k}\right) & =\arg \left(s_{k B}\right)-m \arg \left(s_{k B}\right)+m \arg \left(s_{k T}\right)
\end{aligned}
$$

Such relations are used to construct the generical TBTh pole from the corresponding Butterworth and Thiran poles..

\section{TRANSFER FUNCTION OF THE TRANSITIONAL FIL- TER}

The poles position of the transitional Butterworth-Thiran transfer function is determined by defining a real parameter $m$ that provides a linear variation in the phasorangle of the pole in a relation involving the corresponding Butterworth and Thiran poles

$$
s_{k}=s_{k B}^{1-m} s_{k T}^{m}, \text { for } 0 \leq m \leq 1
$$

where $s_{k}$ is the generical pole of the TBTh transfer function, $s_{k B}$ and $s_{k T}$, the Butterworth and Thiran poles, respectively. The position of $s_{k}$ varies smoothly, as $m$ increases, from $s_{k}=s_{k B}$ for $m=0$, to $s_{k}=s_{k T}$ for $m=1$. The magnitude and the argument of $s_{k}$ are

$$
\begin{aligned}
\left|s_{k}\right| & =\left|s_{k B}\right|^{1-m}\left|s_{k T}\right|^{m} \\
\arg \left(s_{k}\right) & =\arg \left(s_{k B}\right)-m \arg \left(s_{k B}\right)+m \arg \left(s_{k T}\right)
\end{aligned}
$$

Such relations are used to construct the generical TBTh pole from the corresponding Butterworth and Thiran poles.

\section{NUMERICAL RESULTS}

The results of the approximation are shown in the two examples with low pass filters of the eighth and tenth degree.

In the first example the normalized value of the group delay at the origin of all eight degree filters is two samples. The transition filter is achieved for $m=0.6$. In order to get the proposed value of group delay at the origin, the $3 \mathrm{~dB}$ bandwidth of the Butterworth filter is adjusted to the value $\omega_{c} T / \pi=0.3163$.

For $\tau=2$, the $3 \mathrm{~dB}$ bandwidth of the Thiran filter is $\omega_{p} T / \pi=0.2322$, while the bandwidth of transitional filter is slightly wider than the Butterworth filter, and it has a value of $\omega_{p} T / \pi=0.2683$. On the other hand, the maximum value of group delay of the Butterworth filter is 5.33 samples, while the maximum value of group delay of the transition filter is reduced to 3.22 samples.

Table 1. The coefficients of the Butterworth's, Thiran's and TBTh filters for $m=0.6$.

\begin{tabular}{|c|r|r|r|}
\hline Coef. & Butterworth & \multicolumn{1}{c|}{ TBTh } & \multicolumn{1}{c|}{ Thiran } \\
\hline$a_{1}$ & 1.0000000 & 1.0000000 & 1.0000000 \\
\hline$a_{2}$ & -3.3158176 & -2.9408615 & -2.4615386 \\
\hline$a_{3}$ & 5.4638252 & 4.3454928 & 3.0769231 \\
\hline$a_{4}$ & -5.5777755 & -4.0307426 & -2.4615386 \\
\hline$a_{5}$ & 3.7806220 & 2.5096502 & 1.3461539 \\
\hline$a_{6}$ & -1.7209603 & -1.0589553 & -0.5067874 \\
\hline$a_{7}$ & 0.5095335 & 0.2928378 & 0.1266968 \\
\hline$a_{8}$ & -0.0891724 & -0.0481745 & -0.0190522 \\
\hline$a_{9}$ & 0.0070302 & 0.0035898 & 0.0013098 \\
\hline
\end{tabular}

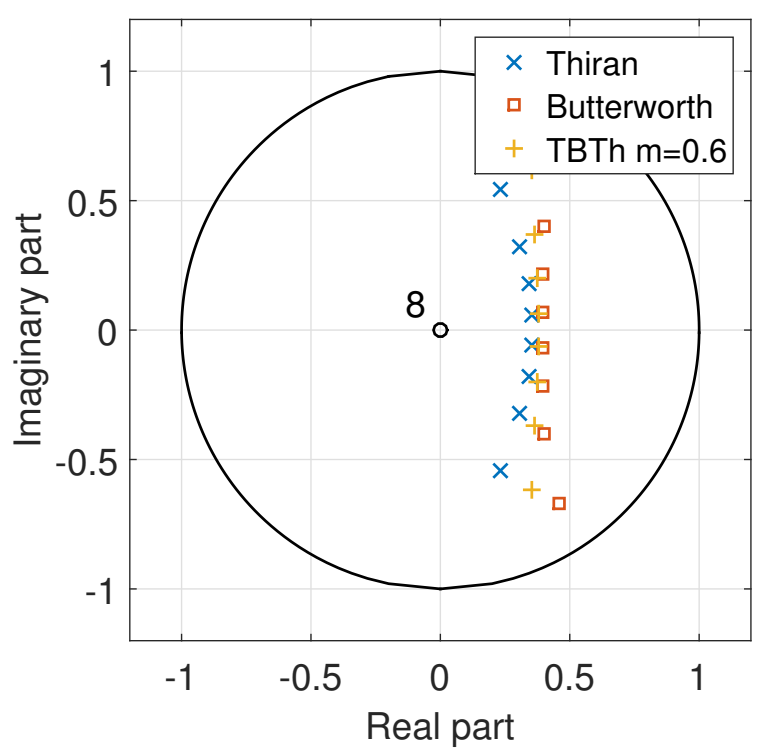

Figure 1. Plot poles and zeros in the z-plane for the 8th degree transfer functions whose coefficients are given in Table 1.

Table 1 displays the coefficients of recursive digital low pass filters of the eighth degree: Butterworth, Thiran and the transitional filter for parameter $m=0.6$. In order to compare the charac- 
teristics of TThB filters with those of other filters, the group delay at $z^{-1}=1$ is taken to be identical, and gain at $z^{-1}=1$ is adjusted to unity. The verification of the transfer functions design given in Table 1 can be performed by the Eq. $(8)^{1}$. The plot poles and zeros in the z-plane of the previously mentioned filters is shown on Figure 1, while their frequency responses can be seen on Figure 2. All filters have a zero of the eighth degree at the origin.

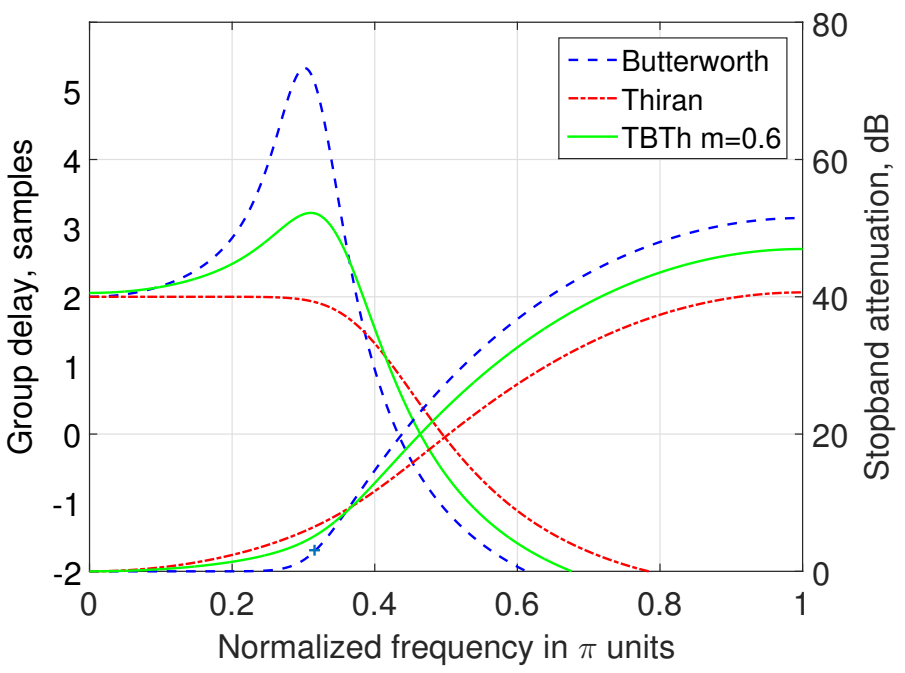

Figure 2. Attenuation and group delay response of the 8th degree filters.

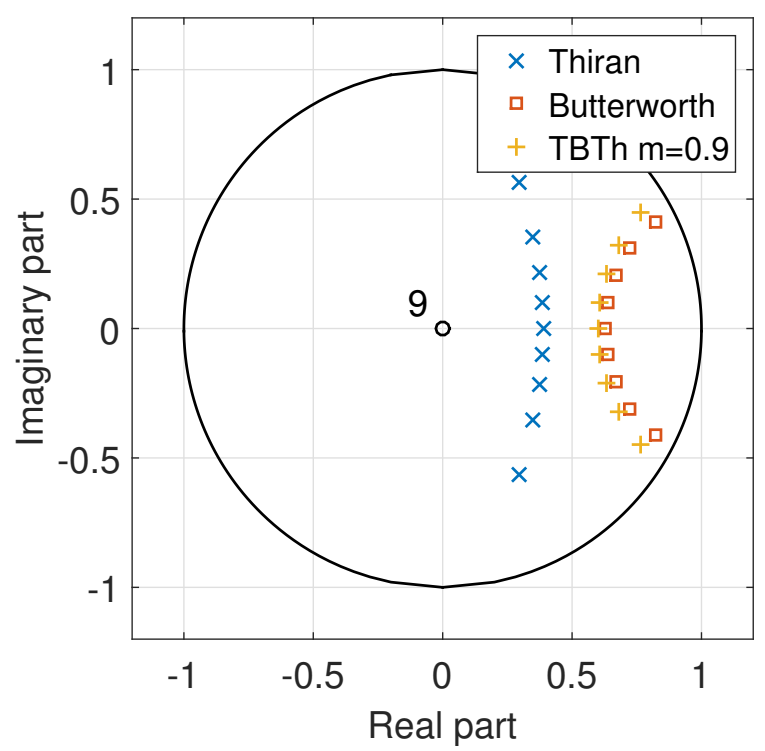

Figure 3. Plot poles and zeros in the $z$-plane for the 10th degree transfer functions.

In the second example the Butterworth, Chebyshev and transitional filters of the tenth degree, when all the filters have the same $3 \mathrm{~dB}$ bandwidth of $\omega_{c} T=0.15 \pi$, are compared. Figure 3 shows the zero and pole position in the $z$-plane, and Figure 4 shows the corresponding frequency responses for all three transfer functions.

1 For all transfer functions given in Table 1 the group delay at origin is $\tau(1)=2$.

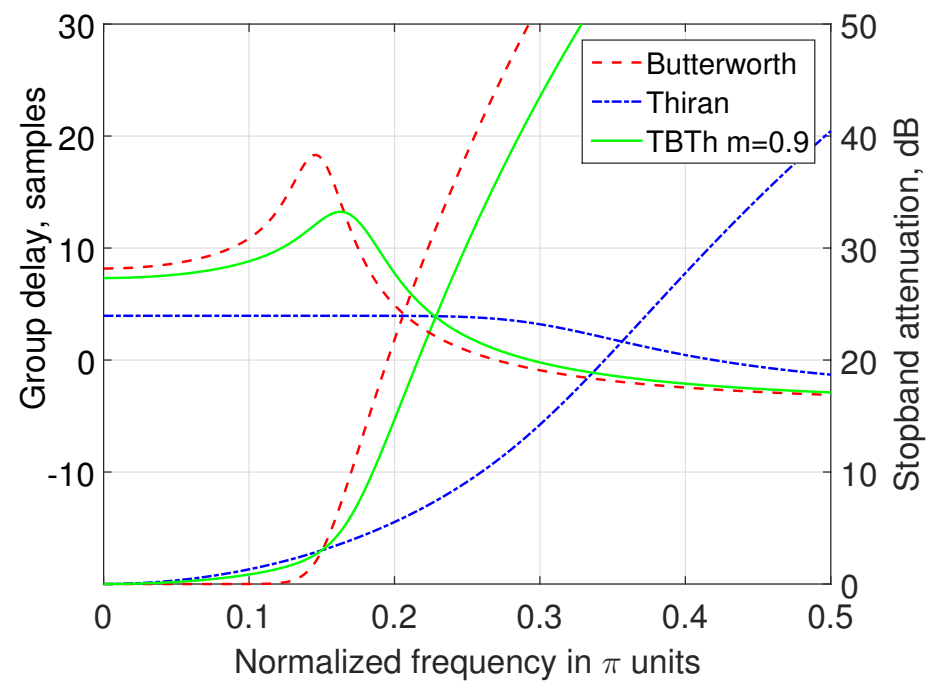

Figure 4. Attenuation and group delay response of the 10th degree filters. The $3-\mathrm{dB}$ cut off frequency is $\omega_{c} T=0.15 \pi$.

Figure 4 shows that for higher values the parameter $m$ achieves a good compromise between stopband attenuation and group delay distortion. On the normalized frequency $\omega=0.2 \pi$ attenuation of the transition filter rises from $5.49 \mathrm{~dB}$ for the TBTh filter, to $11.94 \mathrm{~dB}$. The change in group delay of the transition filter is 5.69 samples, which is considerably lower compared to the changes in group delay of the Butterworth filter which is 11.92 samples.

\section{CONCLUSIONS}

In this paper, a simple method of approximation of transitional Butterworth-Thiran (TBTh) recursive digital filters is proposed. By changing one parameter, the characteristics of these filters change continually from the characteristics of a Butterworth which is maximally flat in the passband filter to those of a Thiran filter which have maximally flat group delay. If we allow for slight deviation of the group delay characteristic from the constant value, the filter stopband attenuation can be significantly increased. In this way, a compromise between the phase distortion in the bandwidth and stop band attenuation can been found.

Keeping in mind that the proposed approximation belongs in the class of auto-regressive (AR) filters, further research includes approximation of the ARMA (Auto Regressive Moving Average) recursive digital filters with maximally flat group delay. In other words, Thirans filter can be expanded using an FIR amplitude corrector, with which it will be possible to preserve the maximally flat group delay of Thirans filter, and improve attenuation in both the passband and in stopband.

\section{REFERENCES}

Aiello, G. \& Angelo, P. 1974. Transitional Legendre Thomson filters. IEEE Transactions on Circuits and Systems, 21(1), pp. 159-162. doi:10.1109/tcs.1974.1083782. 
Attikiouzel, J. \& Dang, T. P. 1978. On transitional ultrasphericalultraspherical filters. Proceedings of the IEEE, 66(6), pp. 703706. doi:10.1109/proc.1978.10996.

Budak, A. \& Aronhime, P. 1971. Transitional ButterworthChebyshev filters. IEEE Transactions on Circuit Theory, 18(3), pp. 413-415. doi:10.1109/tct.1971.1083276.

Johnson, J., Johnson, D., Boudra, P., \& Stokes, V. 1976. Filters using Bessel-type polynomials. IEEE Transactions on Circuits and Systems, 23(2), pp. 96-99. doi:10.1109/tcs.1976.1084174.

Johnson, J., Johnson, D., Perritt, R., \& LaCarna, R. 1979. Transitional rational filters. IEEE Transactions on Circuits and Systems, 26(11), pp. 976-979. doi:10.1109/tcs.1979.1084591.

Lazović, S. M. \& Radmanović, M. D. J. 1975. Transitional Halpern-Thomson filters. Electronics Letters, 11(11), p. 229. doi:10.1049/el:19750174.

Nikolić, S. V. \& Stojanović, V. S. 1996. Transitional ButterworthChebyshev recursive digital filters. International Journal of Electronics, 80(1), pp. 13-20. doi:10.1080/002072196137552.

Papoulis, A. 1958. Optimum Filters with Monotonic Response. Proceedings of the IRE, 46(3), pp. 606-609. doi:10.1109/jrproc.1958.286876.

Peless, Y. \& Murakami. 1957. Analysis and synthesis of tranzitional Butterworth-Thomson filters and bandpass amplifier. RCA Rev., 18(3), pp. 60-94.

Roy, S. \& Varanasi, P. $1978 . \quad$ Transitional Butterworth-Chebyshev filters. Electronics Letters, 14(6), p. 179. doi:10.1049/el:19780119.

Stamenković, N., Stojanović, N., \& Perinić, G. 2018. Group Delay Equalization of Polynomial Recursive Digital Filters in Maxi- mal Flat Sense. Journal of Circuits, Systems and Computers, , p. 1950173. doi:10.1142/s0218126619501731.

Stamenković, N. \& Stojanović, V. 2014. On the design transitional Legendre-Butterworth filters. International Journal of Electronics Letters, 2(3), pp. 186-195. doi:10.1080/00207217.2014.894138.

Stojanović, N., Stamenković, N., \& Stojanović, V. 2014. All-pole recursive digital filters design based on ultraspherical polynomials. Radioengineering, 23(3), pp. 949-953.

Stojanović, V. S. \& Nikolić, S. V. 1993. Direct design of transitional Butterworth-Chebyshev recursive digital filters. Electronics Letters, 29(3), p. 286. doi:10.1049/el:19930195.

Stojanović, V. S. \& Pavlović, V. D. 1979. Transitional Murromaf-Murroer filters. Electronics Letters, 15(12), p. 356. doi:10.1049/el:19790253.

Stojanović, V. V. S. \& V. Nikolić, S. 1998. Direct design of sharp cutoff low-pass recursive digital filters. International Journal of Electronics, 85(5), pp. 589-596. doi:10.1080/002072198133879.

Stojanović, V. S. \& Pavlović, V. D. 1980. Transitional multiplepole transfer functions for active-filter design. Electronics Letters, 16(1), p. 21. doi:10.1049/el:19800018.

Thajchayapong, P., Cheevasuvit, F., \& Manapee, S. 1978. Transitional Butterworth-Chebyshev filters. Electronics Letters, 14(20), p. 680. doi:10.1049/el:19780456.

Thiran, J. P. 1971. Recursive digital filters with maximally flat group delay. IEEE Transactions on Circuit Theory, 18(6), pp. 659-664. doi:10.1109/tct.1971.1083363. 